\title{
Modelo de estaciones de aprendizaje: propuesta para mejorar la enseñanza del inglés en una universidad pública ecuatoriana
}

\author{
Martha Amelia Castillo Noriega \\ https://orcid.org/0000-0002-7867-7463 \\ martha_castillo_noriega@hotmail.com \\ Universidad César Vallejo, Piura - Perú \\ Laura Iliana Mariscal Touzard \\ https://orcid.org/0000-0002-7418-2797 \\ laura.mariscal1978@.gmail.com \\ Universidad de Guayaquil, Guayaquil - Ecuador \\ César Balladares Atoche \\ https://orcid.org/0000-0001-8242-7742 \\ cesarballadares@hotmail.com \\ Universidad César Vallejo, Piura - Perú
}

\section{RESUMEN}

Las estaciones de aprendizaje es un modelo de aprendizaje mixto que puede ser aplicado en cualquier parte y cualquier momento. Con esta premisa este estudio propone un programa de estaciones de aprendizaje basados en recursos digitales gratuitos para mejorar la enseñanza del inglés en los estudiantes de pregrado de una universidad pública. La implementación de este modelo consiste en que los estudiantes roten en un horario establecido con discreción entre las estaciones de aprendizaje en el aula (Staker y Horn, 2012) El diseño del modelo incluyó las cuatro estaciones del modelo: estación del docente, estación individual, estación colaborativa y estación individual / colaborativa, para que tuvieran un equilibrio entre las cuatro habilidades del idioma inglés. El estudio tuvo un enfoque cuantitativo de tipo exploratorio con diseño preexperimental. Los instrumentos fueron validados por expertos y analizados con la prueba de confiabilidad Alfa de Cronbach con valores de 0,776 y 0,925 para la primera y segunda variable, aplicados a una muestra de 120 estudiantes. La valoración cruzada de las dos variables tiene niveles alto de $94.2 \%$ y $81.8 \%$ respectivamente que da apertura para estudios futuros y considerar la implementación este modelo educativo en los cursos de inglés a nivel superior.

Palabras clave: aprendizaje; estaciones; inglés; rotaciones 


\title{
Learning stations model: proposal to improve the teaching of English in an Ecuadorian public university
}

\begin{abstract}
The Station Rotation is a blended learning model that can be applied anywhere and anytime. With this premise, this study proposes a program of learning stations based on free digital resources to improve the English language teaching process in undergraduate students in a state university. The implementation of this model consists of students rotating in a discretely established schedule between the learning stations in the classroom (Staker \& Horn, 2012). This model's design included the four stations of the model: teacher station, individual station, collaborative station, and individual/collaborative station so that they had a balance among the four English language skills. The study had a quantitative exploratory approach with a preexperimental design. Five experts validated the instruments and analyzed them with Cronbach's Alpha reliability test with values of 0.776 and 0.925 for the first and second variables, applied to a sample of 120 students. The cross-assessment of the two variables had high levels of $94.2 \%$ and $81.8 \%$, which leads to future studies and considers the implementation of this educational model in undergraduate English courses.
\end{abstract}

Keywords: EFL; learning; rotations; stations

Artículo recibido: 10 Agosto. 2021

Aceptado para publicación: 07. Setiembre. 2021

Correspondencia: martha_castillo_noriega@hotmail.com

Conflictos de Interés: Ninguna que declarar 


\section{INTRODUCCIÓN}

Este artículo es parte de una tesis doctoral en educación cuyo objetivo general es diseñar un programa de estaciones de aprendizaje, basado en recursos digitales gratuitos, para mejorar la enseñanza del inglés en los estudiantes de pregrado en una universidad pública en Guayquil, Ecuador. Con la implementación del modelo de estaciones de aprendizaje se busca el fortalecimiento de las cuatro destrezas lingüísticas del idioma inglés.

Staker y Horn (2012) sostienen que en el modelo de estaciones de aprendizaje los estudiantes rotan en un horario fijo organizado por el maestro usando las modalidades de aprendizaje en el aula. Una rotación debe al menos ser para el aprendizaje en línea. Las otras estaciones pueden incluir actividades como como instrucción en grupos pequeños o de clase completa, proyectos en grupo, tutoría individual y asignaciones específicas.

De acuerdo con la International Association for K-12 Online Learning, iNACOL (2015), este modelo de rotación de estaciones de aprendizaje fue implementado en las escuelas norteamericanas para todas las materias básicas donde los estudiantes rotaron entre tres estaciones de aprendizaje: instrucción individual, colaborativa y directa cada 20 minutos. Asimismo, la literatura de estudio ha incluido los estudios doctorales han analizado la influencia de este modelo y sus hallazgos constructivos (Jack, 2015; Smalls, 2018; Baker, 2019; y Cargile, 2019) Otros estudios encontraron limitados hallazgos significativos en el rendimiento académico tras la aplicación de las rotaciones (Hiett, 2019 y Mccollum, 2019). La rotación y la habilidad de leer en una estación autónoma permiten a los alumnos mejorar su comprensión lectora (Hui, 2016; Galeas, 2017; Chico, 2019 y Yépez, 2020). Autores también recomendaron la reformulación del plan de estudio (Burgos y Loor, 2017) y el aprendizaje y la asistencia personalizada de los estudiantes en grupos reducidos. (Taylor, 2019) Resultados de la implementación del modelo de estaciones de aprendizaje en aulas de inglés a nivel superior en Brasil muestran la influencia positiva de la implementación teniendo en cuenta aspectos como la variedad de recursos tecnológicos, colaboración entre pares, y rol docente como facilitador de contenido (De Andrade y Rodriguez, 2016; Prudente, 2016; Figueiredo y Valdeni de Lima, 2018, Dos Santos, 2017 y Coutelo de Moraes, Gallindo y Diniz, 2019)

En la universidad pública donde se plantea esta propuesta se observan algunas problemáticas: el elevado número de estudiantes en cada una de las aulas y determinadas carencias presentes en los docentes $\mathrm{y}$, derivado en parte de ello, la insuficiente realización de actividades colaborativas dentro del salón de clase. En consecuencia, no se refuerzan, ni se fomentan las 
cuatro destrezas del idioma: escucha, lectura, escritura, y expresión oral y la interacción áulica se realiza en un ambiente enfocado en el docente y en continuas repeticiones gramaticales. Como causas de estos dos elementos pudiéramos mencionar las dificultades con el equipamiento y manejo eficiente de las TIC, tanto a nivel institucional, como a nivel individual -comprendiendo como tal la triada universidad-docentes-estudiantes-; la ausencia o en ocasiones inaccesibilidad de los docentes a algunos recursos y materiales didácticos, necesarios para el logro y/o perfeccionamiento de un adecuado balance de la práctica de las cuatro destrezas del idioma; el deficiente dominio por parte de algunos docentes, de la didáctica general de la enseñanza del idioma inglés, ya sea en lo concerniente a la enseñanza presencial, como la virtual. Este estudio toma como base la teoría de los Cuatro Aspectros de Nation (1978), la Teoría de adquisición de una segunda lengua de Krashen (1978) y la Teoría de la interdependencia social y aprendizaje cooperativo de Johnson y Johnson (1989)

\section{ESTRATEGIAS METODOLÓGICAS O MATERIALES Y MÉTODOS}

Esta investigación tiene un enfoque cuantitativo de tipo exploratorio con diseño preexperimental. Hernández et al. (2013) sostienen que el tipo exploratorio es conveniente para estudios con problemas poco estudiados e indagadores desde una propuesta innovadora; de igual manera, favorece a identificar conceptos prometedores que permiten la posibilidad para futuros estudios. La población estuvo constituida por 500 unidades (docentes y estudiantes) del programa de inglés del presente período académico. La muestra estuvo constituida por 120 estudiantes y docentes.

\section{Instrumentos de Recolección de Datos}

El instrumento a usar con los estudiantes es una encuesta para conocer sus percepciones sobre la variable independiente: Estaciones de aprendizaje y sus cinco (5) dimensiones. En esta encuesta también se incluyó la variable dependiente: Enseñanza del inglés con sus 15 indicadores, distribuidos entre las cuatro (4) destrezas de inglés.

Este cuestionario tuvo una organización de escala de medición ordinal que, de acuerdo con Arvidsson (2019) consta de valores cuya secuencia es conocida. Esta escala se estipuló con el rango de uno a cuatro con los siguientes valores: (1) Nunca, (2) Pocas veces, (3) Muchas veces y (4) Siempre. 5. La aplicación de la prueba de confiabilidad de Cronbach a la variable Estaciones de Aprendizaje alcanzó 0.776 que se entiende como confiabilidad aceptable. Esta aplicación de prueba a la segunda variable, Enseñanza de inglés, arrojó 0.925 como coeficiente alcanzado correspondiente a una confiabilidad excelente. 


\section{RESULTADOS Y DISCUSIÓN}

\section{a. Formulación de Hipótesis:}

Hi: La distribución de las variables Estaciones de Aprendizaje y Enseñanza de Inglés difieren de la distribución normal. (No es normal)

Ho: La distribución de las variables Estaciones de Aprendizaje y Enseñanza de Inglés no difieren de la distribución normal. (Es normal)

b. Determinación de significancia $\alpha=0.05=5 \%$

Tabla 1. Cuadro de Tablas cruzadas. Estaciones de aprendizaje - Enseñanza del inglés tabulación cruzada

\begin{tabular}{cccccc}
\hline & & \multicolumn{3}{c}{ Enseñanza del idioma inglés } & \multirow{2}{*}{ Total } \\
& & Alto & Medio & Bajo & \\
\hline \multirow{2}{*}{$\begin{array}{c}\text { Estaciones } \\
\text { de } \\
\text { aprendizaje }\end{array}$} & Alto & $67.5 \%$ & $10 \%$ & $5 \%$ & $82.5 \%$ \\
\cline { 2 - 6 } & Medio & 0 & $2.5 \%$ & 0 & $02.5 \%$ \\
\cline { 2 - 6 } & Bajo & $4 \%$ & $5 \%$ & $6 \%$ & $15.0 \%$ \\
\hline Total & $\mathbf{7 1 . 5} \%$ & $\mathbf{1 7 . 5} \%$ & $\mathbf{1 1 \%}$ & $\mathbf{1 0 0 \%}$ \\
\hline
\end{tabular}

La Tabla 1 presenta el cuadro de talas cruzadas entre las dos variables consideradas para este estudio. Aquí se muestra que, tanto para la variable de las estaciones de aprendizaje como para la Enseñanza del inglés, el nivel es alto con el 67.5\%. En cuanto al nivel medio, las dos variables presentan un 5\%. También se observa que ninguna de las dos variables presenta un nivel bajo.

Tabla 2: Estaciones de aprendizaje *Enseñanza del inglés tabulación cruzada

\begin{tabular}{|c|c|c|c|c|c|c|}
\hline & & & \multicolumn{3}{|c|}{ Enseñanza del inglés } & \multirow[t]{2}{*}{ Total } \\
\hline & & & Alto & Medio & Bajo & \\
\hline \multirow[t]{3}{*}{$\begin{array}{l}\text { Estaciones } \\
\text { de } \\
\text { aprendizaje }\end{array}$} & Alto & $\begin{array}{c}\text { \% dentro de } \\
\text { Estaciones de } \\
\text { aprendizaje }\end{array}$ & $81,80 \%$ & $12,10 \%$ & $6,10 \%$ & $100,00 \%$ \\
\hline & & $\begin{array}{c}\text { \% dentro de } \\
\text { Enseñanza del } \\
\text { inglés }\end{array}$ & $94,20 \%$ & $57,10 \%$ & $46,20 \%$ & $82,50 \%$ \\
\hline & Medio & $\begin{array}{c}\text { \% dentro de } \\
\text { Estaciones de } \\
\text { aprendizaje }\end{array}$ & $27,80 \%$ & $33,30 \%$ & $38,90 \%$ & $100,00 \%$ \\
\hline
\end{tabular}




\begin{tabular}{|c|c|c|c|c|c|c|}
\hline & & $\begin{array}{c}\text { \% dentro de } \\
\text { Enseñanza del } \\
\text { inglés }\end{array}$ & $5,80 \%$ & $28,60 \%$ & $53,80 \%$ & $15,00 \%$ \\
\hline & Bajo & $\begin{array}{c}\text { \% dentro de } \\
\text { Estaciones de } \\
\text { aprendizaje }\end{array}$ & & $100,00 \%$ & & $100,00 \%$ \\
\hline & & $\begin{array}{c}\text { \% dentro de } \\
\text { Enseñanza del } \\
\text { inglés }\end{array}$ & & $14,30 \%$ & & $2,50 \%$ \\
\hline \multirow[t]{2}{*}{ Total } & & $\begin{array}{c}\text { \% dentro de } \\
\text { Estaciones de } \\
\text { aprendizaje }\end{array}$ & $71,70 \%$ & $17,50 \%$ & $10,80 \%$ & $100,00 \%$ \\
\hline & & $\begin{array}{c}\text { \% dentro de } \\
\text { Enseñanza del } \\
\text { inglés }\end{array}$ & $100,00 \%$ & $100,00 \%$ & $100,00 \%$ & $100,00 \%$ \\
\hline
\end{tabular}

La Tabla 2 muestra la valoración cruzada de las variables consideradas para este estudio. Se observa que tanto la variable de estaciones de aprendizaje y enseñanza del inglés tienen niveles alto de $94.2 \%$ y $81.8 \%$ respectivamente. De igual manera, se refleja que las dos variables presentan valores similares en el nivel medio. La variable Estaciones de aprendizaje muestra un $33.3 \%$ y la variable de la Enseñanza del inglés un 28.6\%. Finalmente, se evidencia que las dos variables no poseen niveles bajos en esta tabulación cruzada.

Tabla 3: Distribución de frecuencias entre Estaciones de aprendizaje y uso del lenguaje Tablas cruzadas

\begin{tabular}{|c|c|c|c|c|c|}
\hline \multicolumn{6}{|c|}{ Estaciones de aprendizaje * Uso del lenguaje tabulación cruzada } \\
\hline & & & \multicolumn{2}{|c|}{ Uso del lenguaje } & \multirow[t]{2}{*}{ Total } \\
\hline \multirow{7}{*}{$\begin{array}{l}\text { Estaciones de } \\
\text { aprendizaje }\end{array}$} & & & Alto & Medio & \\
\hline & & $\%$ dentro de & & & \\
\hline & Alto & $\begin{array}{l}\text { Estaciones de } \\
\text { aprendizaje }\end{array}$ & $99,00 \%$ & $1,00 \%$ & $100,00 \%$ \\
\hline & & $\begin{array}{c}\text { \% dentro de Uso del } \\
\text { lenguaje }\end{array}$ & $85,20 \%$ & $20,00 \%$ & $82,50 \%$ \\
\hline & & $\%$ dentro de & & & \\
\hline & Medio & $\begin{array}{l}\text { Estaciones de } \\
\text { aprendizaje }\end{array}$ & $77,80 \%$ & $22,20 \%$ & $100,00 \%$ \\
\hline & & $\begin{array}{c}\% \text { dentro de Uso del } \\
\text { lenguaje }\end{array}$ & $12,20 \%$ & $80,00 \%$ & $15,00 \%$ \\
\hline
\end{tabular}




\begin{tabular}{|c|c|c|c|c|c|}
\hline & Bajo & $\begin{array}{l}\% \text { dentro de } \\
\text { Estaciones de } \\
\text { aprendizaje }\end{array}$ & $100,00 \%$ & & $100,00 \%$ \\
\hline & & $\begin{array}{c}\% \text { dentro de Uso del } \\
\text { lenguaje }\end{array}$ & $2,60 \%$ & & $2,50 \%$ \\
\hline \multirow[t]{2}{*}{ Total } & & $\begin{array}{l}\% \text { dentro de } \\
\text { Estaciones de } \\
\text { aprendizaje }\end{array}$ & $95,80 \%$ & $4,20 \%$ & $100,00 \%$ \\
\hline & & $\begin{array}{c}\% \text { dentro de Uso del } \\
\text { lenguaje }\end{array}$ & $100,00 \%$ & $100,00 \%$ & $100,00 \%$ \\
\hline
\end{tabular}

La Tabla 3 muestra la distribución de frecuencias entre las estaciones de aprendizaje y la dimensión del Uso del lenguaje. Se observa que tanto la variable de estaciones de aprendizaje y la dimensión de uso del lenguaje tienen niveles alto de $99 \%$ y $85.2 \%$ respectivamente. En cuanto al nivel medio, se observa que la variable Estaciones de aprendizaje tiene un 20\% mientras que la dimensión del uso del lenguaje tiene un $80 \%$. Finalmente, se evidencia que la dimensión uso del lenguaje no posee niveles bajos en esta tabulación cruzada con la variable de estaciones de aprendizaje. 
Tabla 4: Distribución de frecuencias entre Estaciones de aprendizaje y expresión escrita Tablas cruzadas

\begin{tabular}{|c|c|c|c|c|c|c|}
\hline \multicolumn{7}{|c|}{ Estaciones de aprendizaje * Expresión escrita tabulación cruzada } \\
\hline & & & \multicolumn{2}{|c|}{ Expresión escrita } & \multirow{2}{*}{\multicolumn{2}{|c|}{ Total }} \\
\hline & & & Alto & Medio & & \\
\hline \multirow{6}{*}{$\begin{array}{c}\text { Estaciones de } \\
\text { aprendizaje }\end{array}$} & Alto & $\begin{array}{l}\text { \% dentro de } \\
\text { Estaciones de } \\
\text { aprendizaje }\end{array}$ & $49,50 \%$ & $50,50 \%$ & & $100,00 \%$ \\
\hline & & $\begin{array}{c}\% \text { dentro de } \\
\text { Expresión escrita }\end{array}$ & $94,20 \%$ & $74,60 \%$ & & $82,50 \%$ \\
\hline & Medio & $\begin{array}{l}\text { \% dentro de } \\
\text { Estaciones de } \\
\text { aprendizaje }\end{array}$ & $16,70 \%$ & $77,80 \%$ & $5,60 \%$ & $100,00 \%$ \\
\hline & & $\begin{array}{c}\% \text { dentro de } \\
\text { Expresión escrita }\end{array}$ & $5,80 \%$ & $20,90 \%$ & $100,00 \%$ & $15,00 \%$ \\
\hline & Bajo & $\begin{array}{c}\text { \% dentro de } \\
\text { Estaciones de } \\
\text { aprendizaje }\end{array}$ & & $100,00 \%$ & & $100,00 \%$ \\
\hline & & $\begin{array}{c}\% \text { dentro de } \\
\text { Expresión escrita }\end{array}$ & & $4,50 \%$ & & $2,50 \%$ \\
\hline \multirow[t]{2}{*}{ Total } & & $\begin{array}{l}\text { \% dentro de } \\
\text { Estaciones de } \\
\text { aprendizaje }\end{array}$ & $43,30 \%$ & $55,80 \%$ & $0,80 \%$ & $100,00 \%$ \\
\hline & & $\begin{array}{c}\% \text { dentro de } \\
\text { Expresión escrita }\end{array}$ & $100,00 \%$ & $100,00 \%$ & $100,00 \%$ & $100,00 \%$ \\
\hline
\end{tabular}

La Tabla 4 muestra la distribución de frecuencias entre variable de las estaciones de aprendizaje y la dimensión de expresión escrita. Se observa que tanto la variable de estaciones de aprendizaje y la dimensión de expresión escrita tienen niveles alto de $49.5 \%$ y $94.2 \%$ respectivamente. En cuanto al nivel medio, se observa que la variable Estaciones de aprendizaje tiene un $77.8 \%$ mientras que la dimensión de expresión escrita tiene un $20.9 \%$. Finalmente, se evidencia que tanto, la dimensión expresión escrita como la variable de estaciones de aprendizaje, no poseen niveles bajos en esta tabulación cruzada. 
Tabla 5: Distribución de frecuencias entre Estaciones de aprendizaje y comprensión auditiva - Tablas cruzadas

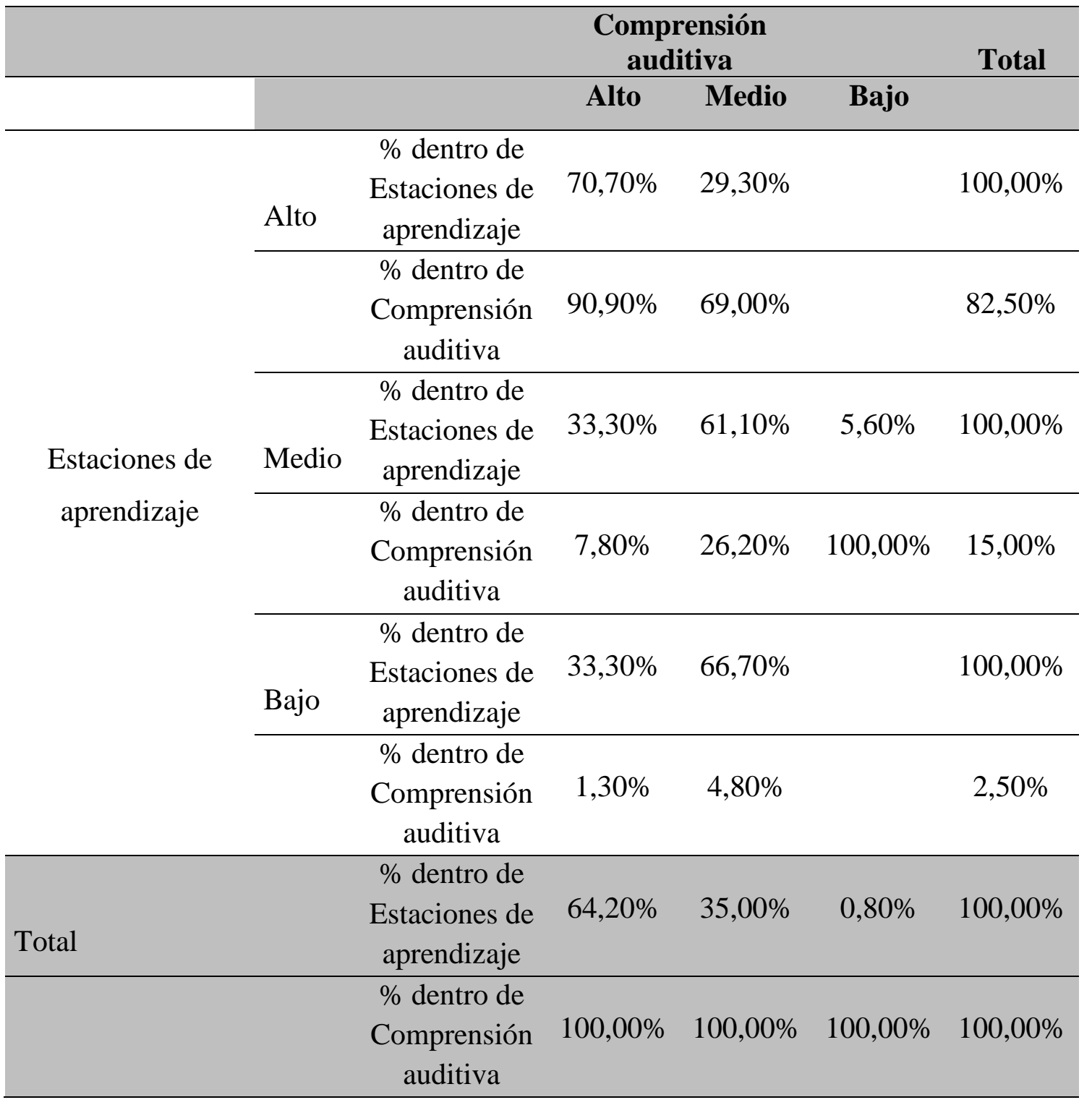

La Tabla 5 muestra la distribución de frecuencias entre las estaciones de aprendizaje y la dimensión de la comprensión auditiva. Se observa que tanto la variable de estaciones de aprendizaje y la dimensión de la comprensión auditiva tienen niveles alto de 70.7\% y 90.9\% respectivamente. En cuanto al nivel medio, la variable Estaciones de aprendizaje tiene un $61.1 \%$ mientras que la dimensión de la comprensión auditiva tiene un 26.2\%. Finalmente, se refleja 
que en esta tabulación cruzada no existen niveles bajos entre la dimensión de la comprensión auditiva y la variable de estaciones de aprendizaje.

Tabla 6: Distribución de frecuencias entre Estaciones de aprendizaje y expresión oral Tablas cruzadas

\begin{tabular}{|c|c|c|c|c|c|c|}
\hline & & & Expres & ón oral & & Total \\
\hline & & & Alto & Medio & Bajo & \\
\hline & Alto & $\begin{array}{l}\text { \% dentro de } \\
\text { Estaciones de } \\
\text { aprendizaje }\end{array}$ & $66,70 \%$ & $33,30 \%$ & & $100,00 \%$ \\
\hline & & $\begin{array}{l}\text { \% dentro de } \\
\text { Expresión oral }\end{array}$ & $94,30 \%$ & $68,80 \%$ & & $82,50 \%$ \\
\hline $\begin{array}{c}\text { Estaciones } \\
\text { de }\end{array}$ & Medio & $\begin{array}{l}\text { \% dentro de } \\
\text { Estaciones de } \\
\text { aprendizaje }\end{array}$ & $22,20 \%$ & $66,70 \%$ & $11,10 \%$ & $100,00 \%$ \\
\hline aprendizaje & & $\begin{array}{l}\% \text { dentro de } \\
\text { Expresión oral }\end{array}$ & $5,70 \%$ & $25,00 \%$ & $100,00 \%$ & $15,00 \%$ \\
\hline & Bajo & $\begin{array}{l}\text { \% dentro de } \\
\text { Estaciones de } \\
\text { aprendizaje }\end{array}$ & & $100,00 \%$ & & $100,00 \%$ \\
\hline & & $\begin{array}{l}\text { \% dentro de } \\
\text { Expresión oral }\end{array}$ & & $6,30 \%$ & & $2,50 \%$ \\
\hline Total & & $\begin{array}{l}\text { \% dentro de } \\
\text { Estaciones de } \\
\text { aprendizaje }\end{array}$ & $58,30 \%$ & $40,00 \%$ & $1,70 \%$ & $100,00 \%$ \\
\hline & & $\begin{array}{l}\% \text { dentro de } \\
\text { Expresión oral }\end{array}$ & $100,00 \%$ & $100,00 \%$ & $100,00 \%$ & $100,00 \%$ \\
\hline
\end{tabular}

La Tabla 6 muestran la distribución de frecuencias entre las estaciones de aprendizaje y la dimensión de la expresión oral. Se observa que tanto la variable de estaciones de aprendizaje y la dimensión de la expresión oral tienen niveles alto de 66,70\% y $94.3 \%$ respectivamente. En cuanto al nivel medio, la variable Estaciones de aprendizaje tiene un $66.7 \%$ mientras que la dimensión de la expresión oral tiene un 25\%. Finalmente, se confirma que en esta tabulación cruzada no existen niveles bajos entre la dimensión de la expresión oral y la variable de estaciones de aprendizaje.

\section{CONCLUSIÓN O CONSIDERACIONES FINALES}

Los altos valores del nivel de logro del modelo reflejan una aplicación básica -pero positiva- de los cinco componentes del modelo en diferentes momentos. Se confirma que el intervalo de 
frecuencia de la variable estaciones de aprendizaje arrojó un 82.5\% de la muestra se ubicó en un nivel de logro alto. El rango medio estuvo representado en un $15 \%$, mientras que el $2.5 \%$ reflejó un intervalo de frecuencia bajo.

El alto valor del nivel de logro correspondiente a la variable Enseñanza del idioma inglés en que se resalta en el $71.7 \%$ de los estudiantes quien obtuvieron ese alto intervalo, seguido del $17.5 \%$ que representó un rango medio, con un $10.8 \%$ de intervalo bajo. Los resultados demuestran una identificación de las cuatro destrezas del idioma que le permite conocer los momentos de adquisición (lectura, comprensión auditiva) y producción (oral o escrita). Esta identificación de destrezas favorece la aplicación del modelo con una actividad por estación donde se puedan diseñar actividades para reforzar cada habilidad del lenguaje.

Se recomienda socializar la propuesta de este estudio con el Vicerrectorado Académico de esta institución superior, de manera que se pueda mejorar la enseñanza del inglés.

Se recomienda a los docentes planificar sus estaciones de aprendizaje cuidadosamente y tener un objetivo de lección claro, teniendo en consideración el recurso gratuito disponible, sus limitaciones y las del aula. Cabe recordar que el éxito de las estaciones de aprendizaje dependerá de la creatividad del docente de inglés en distribuir las cuatro estrategias del idioma de manera balanceada, lo que permita la mejor adquisición de información, con el docente como mediador, diseñador y curador del contenido que se presenta en su cátedra.

En cuanto a los estudiantes se recomienda reforzar su autonomía para encontrar soluciones cuando se presente alguna dificultad con la actividad y como grupo deban resolverla. Parte del aprendizaje consiste en aceptar los errores como parte del proceso de aprendizaje y no tomarlos como fracaso.

\section{LISTA DE REFERENCIAS}

Arvidsson, R. (2019). Sobre el uso de escalas de puntuación ordinales en la evaluación del ciclo de vida social. The International Journal of Life Cycle Assessment. 24:604-606. https://doi.org/10.1007/s11367-018-1557-2

Baker, D. (2019). Un estudio de caso único de aprendizaje mixto en una escuela secundaria de Colorado. Tesis para optar por el grado académico de Doctor en Educación de Concordia University-Portland. Oregon. Estados Unidos.

Burgos, A. y Loor, M. (2017). Influencia de la práctica lectora intensiva en el desarrollo de las habilidades de estudiantes de pregrado de la universidad pública ecuatoriana. Tesis 
para optar el grado académico de Master en la enseñanza del inglés como lengua extranjera. Escuela Superior Politécnica del Litoral. Guayaquil. Ecuador

Cargile, L. (2019). El impacto del aprendizaje mixto con Khan Academia y proyectos sobre la motivación en un aula de matemáticas. Tesis para optar por el grado académico de Doctor en Educación de Cincinnati University. Ohio. Estados Unidos

Chico, L. (2019). Cuentos en inglés para comprensión lectora. Tesis para optar por el título académico de Magister en la Enseñanza del Idioma Inglés como Lengua Extranjera de la Universidad Técnica de Ambato. Ambato. Ecuador.

Coutelo de Moraes, A., Galindo, L. y Góis Diniz, M. (2019). Aprendizaje combinado y modelo de rotación: enseñanza de una lengua extranjera a adultos. https://www.hltmag.co.uk/aug19/blended-learning

De Andrade, M., y Rodríguez de Souza, P. (2016). Modelos híbridos de rotación de enseñanza: Estaciones de trabajo y aula invertida.: https://etech.sc.senai.br/edicao01/article/view/773

Dos Santos, H. (2017). Aprendizaje mixto en la enseñanza del inglés como lengua extranjera: Un estudio de caso con profesores en formación. https://wp.ufpel.edu.br/ppgl/files/2018/11/VERS\%C3\%83O-FINALDISSERTA\%C3\%87\%C3\%83O-HELENA-DOS-SANTOS-KIELING.pdf

Figueriedo, M.,y Valdeni de Lima, J. (2019). Participación activa en el aula a través de espacios híbridos: Un estudio en educación técnica integrada. https://www.researchgate.net/publication/332941000

Galeas, G. (2017). Leer para aprender: Comprender la lectura dentro de la instrucción de vocabulario integrado en estudiantes de inglés como lengua extranjera. Tesis para optar el grado académico de Master en la enseñanza del inglés como lengua extranjera. Escuela Superior Politécnica del Litoral. Guayaquil. Ecuador.

Hernández R., Baptista, P., Fernandez C. y Méndez S. (2013). Metodología De La Investigación (6ta Edición) MC Graw Hill Education

Hiett, B. (2019). Un examen del aprendizaje combinado y el aula tradicional utilizando puntuaciones de rendimiento. Tesis para optar por el grado académico de Doctor en Filosofía y Tecnología Educativa de Walden University. Minneapolis. Estados Unidos.

Hui, M. (2016). Un maestro desarrolló un modelo de aprendizaje mixto sobre la construcción de habilidades de comprensión de lectura para apoyar el desempeño en todo el plan de 
estudios. Tesis para optar por el grado académico de Doctor en Educación y Psicología de Universidad de Navarra. Pamplona. España.

International Association for K-12 Online Learning. (2015). Blending Learning: The Evolution of Online and Face-to-Face Education from 2008-2015

Jack, L. (2015). Un análisis de la implementación y el efecto de rompecabezas en estrategias de aprendizaje cooperativo en estudiantes de noveno grado: Rendimiento en Algebra 1. Tesis para optar por el grado académico de Doctor de Filosofía en Ciencias, Matemáticas y Educación de Southern University. Louisiana. Estados Unidos.

Johnson, D. W., y Johnson, R. T. (1989). Una historia de éxito en psicología educativa: teoría de la interdependencia social y aprendizaje cooperativo. Educational researcher, 38(5), 365-379.

Krashen. S. (1978). Teoría de la adquisición de una segunda lengua. Prentice-Hall International, 1978

McCollum, T. (2019). Una comparación de un aula de aprendizaje mixto de rotación de estaciones con un aula tradicional utilizando NWEA MAP. Tesis para optar el grado académico de Doctor en Educación de McKendree University. Illinois.

Nation, P. (2007) Los cuatro aspectos, innovación en el aprendizaje y la enseñanza de idiomas, 1:1, 2-13, DOI: $10.2167 /$ illt039.0

Smalls, L. (2018). Efectividad del modelo de estaciones de aprendizaje en el entorno de la escuela intermedia para aumentar las calificaciones de los estudiantes en las evaluaciones del aula y del distrito. Tesis para optar por el grado académico de Doctor en Educación de Universidad de Brenau. Georgia. Estados Unidos.

Staker, H. y Horn, M. (2012). Classifying K-12 Blended Learning. https://www.christenseninstitute.org/wp-content/uploads/2013/04/Classifying-K-12blended-learning.pdf

Taylor, B. (2019). Un estudio de casos de experiencias de profesores de secundaria con aprendizaje personalizado. Tesis para optar por el grado académico de Doctor en Filosofía de Mercer University. Atlanta. Estados Unidos 